\title{
Diagnóstico tardio de malária em área endêmica de dengue na extra- Amazônia brasileira: experiência recente de uma unidade sentinela no Estado do Rio de Janeiro
}

\author{
Delayed diagnosis of malaria in a dengue endemic area in the Brazilian extra-Amazon: recent \\ experience of a malaria surveillance unit in State of Rio de Janeiro
} \begin{abstract}
Cláudio Tadeu Daniel-Ribeiro ${ }^{2,5}$ e Patrícia Brasil ${ }^{1,2,6}$
\section{RESUMO}

Introdução: A letalidade da malária na região extra-amazônica é cerca de 80 vezes maior do que na Amazônia, que concentra 99,8\% dos casos do país. Em áreas de transmissão de dengue, como o Rio de Janeiro, o atraso no diagnóstico e tratamento da malária dos pacientes com febre, provenientes de áreas endêmicas de malária, pode ser, entre outros fatores, devido à confusão entre o diagnóstico das duas doenças pelos generalistas da rede de assistência médica. Neste trabalho, apresentamos as consequências do atraso diagnóstico em três pacientes com malária por Plasmodium falciparum; $P$. malariae e $P$. vivax, que, após o périplo habitual para tratamento de dengue, procuraram a nossa instituição onde foram corretamente diagnosticados e submetidos aos tratamentos adequados. Métodos: Descrição de três casos de malária diagnosticada tardiamente e encaminhados ao IPEC/ FIOCRUZ, entre os anos de 2007 e 2008. Resultados: uma brasileira proveniente de Moçambique, primo-infectada por P.falciparum, com malária diagnosticada após 6 dias do início da febre, morreu com malária cerebral e choque. Outro paciente com malária por $P$. malariae teve um curso grave e prolongado, mas ficou curado após o tratamento específico. A terceira paciente diagnosticada tardiamente apresentou malária por $P$. vivax adquirida na região de Mata Atlântica no Estado do Rio. Conclusões: Os profissionais de saúde do Rio devem ser treinados para aperfeiçoar a vigilância e o tratamento oportuno da malária e evitar desfechos mórbidos e fatais. Sugere-se que uma investigação de focos de malária autóctone em áreas de mata no estado seja realizada.

Palavras-chaves: Malária. Dengue. Diagnóstico diferencial. Vigilância.
\end{abstract}

Anielle de Pina Costa ${ }^{1,2}$, Clarisse da Silveira Bressan ${ }^{1,2}$, Renata Saraiva Pedro ${ }^{2,3}$, Rogério Valls-de-Souza ${ }^{1}$, Sidnei da Silva ${ }^{2,4}$, Patrícia Rosana de Souza ${ }^{1}$, Lusiele Guaraldo ${ }^{2,3}$, Maria de Fátima Ferreira-da-Cruz ${ }^{2,5}$,

\begin{abstract}
Introduction: The mortality of malaria in the extra-Amazon region is about 80 times higher than in the Amazon region, where malaria is concentrated (99.8\% of cases). In areas of dengue transmission, delay in the diagnosis and treatment of malaria in patients with fever who reside in areas of malaria transmission can be due to the confusion between the clinical diagnoses of both diseases by nonspecialist doctors, among other factors. This work presents some of the consequences of delayed diagnosis in three patients with malaria by Plasmodium falciparum, P. malariae and P. vivax, who, after following the usual route for Dengue treatment, sought our institution, where they were correctly diagnosed and adequately treated. Methods: Description of three cases of malaria with delayed diagnosed malaria referred to the Outpatient Clinic for Acute Febrile Diseases, IPEC/FIOCRUZ-RJ, between 2007 and 2008. Results: A Brazilian from Mozambique, primo-infected with P. falciparum was diagnosed with malaria six days after the onset of fever and died of cerebral malaria and shock. Another patient with P. malariae malaria presented a severe and prolonged course, but was cured after specific treatment. A third patient, with delayed diagnosis of P. vivax malaria, acquired it in the Atlantic Forest region in the State of Rio. Conclusions: Health professionals from non-endemic areas for malaria should be trained to optimize the surveillance and early treatment of malaria and prevent morbid and fatal outcomes. An investigation of outbreaks of autochthonous malaria in the State of Rio de Janeiro is suggested.
\end{abstract}

Key-words: Malaria. Dengue. Diagnosis. Surveillance.

1. Serviço de Doenças Febris Agudas, Instituto de Pesquisa Clínica Evandro Chagas, Fundação Oswaldo Cruz, Rio de Janeiro, RJ. 2. Centro de Pesquisa, Diagnóstico e Treinamento em Malária, Fundação Oswaldo Cruz, Rio de Janeiro, RJ. 3. Serviço de Farmacovigilância, Instituto de Pesquisa Clínica Evandro Chagas, Fundação Oswaldo Cruz, Rio de Janeiro, RJ. 4. Serviço de Parasitologia, Instituto de Pesquisa Clínica Evandro Chagas, Fundação Oswaldo Cruz, Rio de Janeiro, RJ. 5. Laboratório de Pesquisa em Malária, Instituto Oswaldo Cruz, Fundação Oswaldo Cruz, Rio de Janeiro, RJ. 6. Serviço de Vigilância em Saúde, Instituto de Pesquisa Clínica Evandro Chagas, Fundação Oswaldo Cruz, Rio de Janeiro, RJ.

Endereço para correspondência: Dra Patrícia Brasil. Serviço de Doenças Febris Agudas e Vigilância em Saúde/IPEC/FIOCRUZ. Av Brasil 4365, Manguinhos, 21040-900 Rio de Janeiro, RJ.

Telefax: $55213865-9554$

e-mail: patrícia.brasil@ipec.fiocruz.br

Recebido para publicação em 11/02/2010

Aceito em 27/07/2010

\section{INTRODUÇÃO}

A malária é a endemia parasitária mais prevalente no mundo, afetando cerca de 250 milhões de pessoas em mais de 109 países, especialmente no continente africano, Ásia e América Central ${ }^{1}$. No Brasil, a doença é endêmica na região amazônica com transmissão esporádica em outras regiões devido à presença de vetores em mais de $80 \%$ do território nacional ${ }^{2,3}$. Embora a tríade clássica da malária seja constituída por calafrios, febre intermitente e cefaléia ${ }^{4-6}$, os sintomas da fase inicial - mal estar, náuseas, tonturas, cansaço, mialgia, febre contínua e sudorese $\mathrm{e}^{5,7}$ - são inespecíficos e comuns à maioria das síndromes febris agudas, o que pode confundir profissionais de saúde e retardar o seu diagnóstico ${ }^{5}$. Tal retardo, comum em áreas onde a doença não é endêmica, pode resultar em doença grave e óbito, também proporcionalmente mais comum na região extra-amazônica. Assim, o diagnóstico e tratamento precoces da malária são fundamentais para melhorar o prognóstico e diminuir a letalidade da doença ${ }^{8-12}$.

O dengue é uma arbovirose transmitida por mosquitos do gênero Aedes, especialmente pelo Aedes aegypti no Brasil ${ }^{1,13}$. Dois e meio bilhões de pessoas vivem em área de risco para contrair dengue no mundo ${ }^{14} \mathrm{e}$, anualmente, ocorrem $50 \mathrm{e}$ 100 milhões de infecções ${ }^{15,14}, 500.000$ casos de febre hemorrágica da dengue (FHD) e 21.000 óbitos $^{14}$. No Brasil, desde a década de 1980 vêm ocorrendo epidemias pelo vírus dengue (DENV) nos principais centros urbanos do país, com cerca de três milhões de casos, com aumento na gravidade ao longo dos $\operatorname{anos}^{16}$. O Rio de Janeiro viveu recentemente uma alarmante epidemia, onde de janeiro a agosto de 2008 foram notificados 250.200 casos $^{17}$ com 123 óbitos, sendo 45 por febre hemorrágica do dengue (FHD), 22 por síndrome do choque do dengue (SCD) e 56 por dengue com complicações, sendo $42 \%$ em menores de 15 anos $^{18}$. A infecção pelo vírus 
da dengue pode provocar um amplo espectro de manifestações, desde formas assintomáticas ou pouco sintomáticas, autolimitadas, até apresentações graves, por vezes fatais ${ }^{13,14}$. As manifestações mais frequentes do dengue clássico são febre, cefaléia, dor retro-orbital, mialgias, artralgias, astenia e prostração $0^{7,13,14,19}$. Manifestações gastrintestinais e linfadenopatias podem ocorrer ${ }^{13,14}$. A febre tem duração média de cinco a sete dias ${ }^{7,13,14}$.

Neste trabalho, destacamos a dificuldade diagnóstica ${ }^{8}$ e a importância da indicação precoce do tratamento ${ }^{8-12,20}$ da malária em uma área endêmica de dengue na região extra-amazônica.

\section{MÉTODOS}

Estudo descritivo de pacientes com malária atendidos no Serviço de Doenças Febris Agudas do IPEC/FIOCRUZ, localizado no Rio de Janeiro, no período entre 2007 e 2008.

\section{Critérios de inclusão}

Foram selecionados três pacientes com malária encaminhados ao Serviço com diagnóstico clínico de dengue.

\section{Variáveis de interesse}

Dados sociodemográficos, história de exposição, anamnese, exame físico, hemograma, provas de função hepática e renal, evolução clínica e desfecho (cura ou óbito).

\section{Diagnóstico laboratorial}

Foi realizada pesquisa direta de plasmódio em gota espessa e distensão sanguínea, pelo Laboratório de Parasitologia do IPEC/ FIOCRUZ, seguindo as recomendações do Ministério da Saúde ${ }^{21}$ e diagnóstico molecular através do método da reação em cadeia de polimerase (PCR) convencional espécie - específica para P. viva ${ }^{22} \mathrm{e}$ P. falciparum ${ }^{23}$ realizados no Laboratório de Pesquisas em Malária do Instituto Oswaldo Cruz (LPM/IOC/FOCRUZ). O diagnóstico de P. malariae foi feito por distensão sanguínea. Foram coletados $9 \mathrm{~mL}$ de sangue por punção venosa sem anticoagulante para realização de bioquímica (transaminases, bilirrubinas, uréia e creatinina) e $8 \mathrm{~mL}$ de sangue com EDTA para pesquisa direta de plasmódio e PCR, além de hemograma completo, de cada paciente.

\section{Ética}

O projeto foi submetido e aprovado pelo Comitê de Ética em Pesquisa do IPEC/FIOCRUZ, sob o número 0045.0.009.000-9.

\section{RESULTADOS}

\section{Descrição clínica}

Mulher, parda, 40 anos, missionária religiosa, previamente hígida, natural do RJ, imunizada para febre amarela. Retornou ao Rio de Janeiro em 23 de fevereiro de 2007 proveniente de Moçambique, onde trabalhou por 14 dias como voluntária. Referia história iniciada em 1 de março de 2007 com febre, calafrios, cefaléia, hematúria, mialgia e astenia. Procurou atendimento médico na rede privada onde recebeu diagnóstico clínico de dengue, foi hidratada por via venosa e recebeu antitérmico. Não foi feita pesquisa de plasmódio. Na manhã de 7 de março, procurou o ambulatório de DFA do IPEC, com febre contínua, deambulando, lúcida, orientada no tempo e no espaço, hipocorada $(1+/ 4+)$, ictérica $(1+/ 4+)$, eupnéica, com hepatoesplenomegalia. Foi admitida no setor de urgência e administrado artesunato endovenoso em dose de ataque
$(2,4 \mathrm{mg} / \mathrm{kg}$ - ataque, seguido de $1,2 \mathrm{mg} / \mathrm{kg}$ em 4 e 24 horas $)$ associado à clindamicina $(600 \mathrm{mg}, 8 / 8 \mathrm{~h})$. Evoluiu com redução súbita do nível de consciência, espasmos musculares, vômito em jato. Feita uma tomografia computadorizada de crânio que revelou edema cerebral difuso. O hemograma mostrou plaquetopenia (23.000/ $\mathrm{mm}^{3}$ ), hematócrito de $28 \%$, e hemoglobina de $9,4 \mathrm{mg} / \mathrm{dl}, 12.500$ leucócitos e 15 bastões. O exame parasitológico evidenciou $48 \%$ das hemácias parasitadas por P. falciparum (parasitas $>200.000$ / $\mathrm{mm}^{3}$ ). Realizada transferência da paciente para a UTI onde foram iniciados procedimentos de ventilação mecânica, aminas vasoativas, transfusão de concentrado de hemácias, plaquetas e plasma devido a sangramento difuso. Doze horas após o início da terapia, a parasitemia caiu para $30.720 \mathrm{p} / \mathrm{mm}^{3}$, e a paciente continuava em coma profundo, com rigidez de nuca $(3+/ 4+)$, sinal de Brudzinsky positivo, extremidades frias e perfusão capilar lentificada. A paciente morreu em $24 \mathrm{~h}$ da internação com choque refratário.

Homem, branco, 36 anos, cinegrafista, natural do RJ, com diabetes tipo II, imunizado para febre amarela e hepatite B, retornou ao Rio de Janeiro em março de 2007 após seis semanas de trabalho na Cidade de Barcelos no alto Rio Negro (AM). Em 21 de setembro, apresentou mal-estar seguido de febre alta $\left(40^{\circ} \mathrm{C}\right)$ com calafrios, mialgia, tosse seca e diarréia. Em 26 de setembro, foi internado em hospital privado com anemia e insuficiência renal aguda, onde recebeu terapia de suporte em função da suspeita clínica diagnóstica de dengue. A sorologia ( $\operatorname{IgM}$ ) para dengue foi negativa e não foi feita pesquisa para plasmódio. Sete dias depois, em 9 de outubro, procurou o ambulatório de Doenças Febris Agudas (DFA) do IPEC com queixa de calafrios e febre do tipo quartã. Ao exame físico, apresentava-se em mau estado geral, hipocorado $(2+/ 4+)$, ictérico $(2+/ 4+)$ e com hepatoesplenomegalia. Apresentava anemia, sem plaquetopenia $(\mathrm{Hb}$ $9,3 \mathrm{~g} / \mathrm{dl}, \mathrm{Ht} 29,3 \%$ plaquetas $222.000 / \mathrm{mm}^{3}$, leucócitos $\left.4.430 / \mathrm{mm}^{3}\right)$, e a distensão do sangue periférico evidenciou P. malariae (parasitemia $1.040 \mathrm{p} / \mathrm{mm}^{3}$ ), além de hemácias normocrômicas e normocíticas. Foi prescrita cloroquina, durante três dias. Houve melhora clínica e queda da parasitemia $\left(440 \mathrm{p} / \mathrm{mm}^{3}\right)$ em $24 \mathrm{~h}$. Em uma semana, o paciente encontrava-se assintomático, com exame parasitológico negativo e normalização do hemograma.

Mulher, branca, 28 anos, bióloga, natural do Rio de Janeiro, com relato de entradas sucessivas e pernoites em área de Mata Atlântica (Serra dos Órgãos, RJ), no período de 01/02 a 12/03/08, para realização de trabalho de campo. História iniciada em 4 de março com dor abdominal, diarréia líquida (>10 episódios/dia) autolimitada, cefaléia intensa, mialgia e parestesia de extremidades. Em 48 horas, evoluiu com febre do tipo terçã e calafrios acompanhados de artralgia, além de um episódio de hematúria e epistaxe. Em 17 de março, após seguidos atendimentos hospitalares, com quadro clínico atribuído à infecção pelo vírus do dengue, procurou o ambulatório de DFA do IPEC. Ao exame físico, apresentava-se em bom estado geral, afebril, anictérica, hidratada, hipocorada $(1+/ 4+)$, sem exantemas ou petéquias, com esplenomegalia. Os exames laboratoriais estavam inalterados, sem anemia ou plaquetopenia ( $\mathrm{Ht} 37 \%, \mathrm{Hb}$ 12,7, leucócitos $4.800 / \mathrm{mm}^{3}$, plaquetas 182.000$)$. A pesquisa direta em sangue periférico (gota espessa) evidenciou P. vivax $\left(80\right.$ parasitas $\left./ \mathrm{mm}^{3}\right)$. A paciente negava viagem à área endêmica de malária, uso de drogas endovenosas ou hemotransfusões. Foram prescritas cloroquina por três dias e primaquina por sete dias. Em $48 \mathrm{~h}$, houve negativação da parasitemia e remissão dos sintomas. Na terceira semana, apresentava-se em bom estado geral; porém, mantinha a esplenomegalia, que desapareceu na $6^{\mathrm{a}}$ semana após o início do tratamento. 


\section{DISCUSSÃo}

As espécies que causam malária no Brasil são Plasmodium vivax - que responde por cerca de $85 \%$ dos casos registrados, $P$. falciparum - que causa aproximadamente de $15 \%$ deles, e P. malariae - responsável por uma diminuta percentagem dos $\operatorname{casos}^{2,3,24}$. Não há transmissão de P. ovale no Brasil, e os poucos registros são importados de países africanos ${ }^{7}$. A febre alta contínua ou em paroxismos a cada $48 \mathrm{~h}$, febre terçã $\tilde{a}^{5}(P$. vivax e P. falciparum) ou $72 \mathrm{~h}$, febre quartã ${ }^{5}$ (P. malariae) é, habitualmente, acompanhada de calafrios, intensa queda do estado geral, astenia e mialgia ${ }^{5,710}$. Em geral, ocorre também prostração, cefaléia e alterações laboratoriais como elevação de bilirrubinas e distúrbios nos fatores da coagulação ${ }^{25}$. O padrão-ouro para o diagnóstico é o exame direto do sangue periférico com realização de gota espessa, mais sensível para o diagnóstico da infecção do que a distensão sanguínea, na qual, entretanto se identifica mais facilmente a espécie de Plasmodium ${ }^{9-12}$.

Este trabalho ilustra as dificuldades da abordagem do paciente febril em uma área endêmica de dengue, na região extra-Amazônica. Os três pacientes descritos foram atendidos inicialmente em hospitais privados por médicos generalistas ou socorristas que, sem a atenção devido à história de deslocamentos para áreas endêmicas, e diante da inespecificidade da apresentação clínica da doença, não consideraram a malária no diagnóstico diferencial do quadro febril agudo, e atribuíram o quadro dos três pacientes, à possível infecção pelo vírus do dengue. Embora os paroxismos cíclicos da febre, com periodicidade terçã, na malária por $P$. vivax e quartã na malária por P. malariae sejam característicos da apresentação clínica e estivessem presentes em dois dos três pacientes, a hipótese diagnóstica de malária só foi evocada pelos especialistas em doenças infecciosas. Além disso, a persistência da febre após sete dias de evolução, não usual no dengue, mas comum na malária não tratada, também passou despercebida. Achados do exame físico, como palidez e esplenomegalia característicos da malária e pouco usuais no dengue, poderiam sugerir o diagnóstico de malária se associados à história de deslocamento para área endêmica ao profissional bem treinado.

O tempo decorrido entre o início dos sintomas e o diagnóstico da malária foi de seis dias no caso de malária por P.falciparum, 13 dias no de malária por $P$. vivax, e 18 dias na malária por $P$. malariae, ilustrando como o atraso diagnóstico superior a 48 horas pode ser fator de risco associado ao óbito na malária por $P$. falciparum, de forma diferente da malária causada pelas demais espécies, aparentemente benignas.

A trombocitopenia, frequentemente atribuída à febre hemorrágica do dengue (FHD), em pacientes febris atendidos em Unidades de Saúde do Rio de Janeiro, implica, assim, em uma superestimativa da suspeita de dengue por ser inadvertidamente utilizada como preditiva diagnóstica de FHD, situação da qual ela não é uma alteração laboratorial exclusiva ${ }^{25}$.

A anemia foi comum à malária grave por $P$. falciparum è malária por P. malariae, provavelmente por mecanismos de fisiopatologia distintos. Na malária por P.falciparum, ela teria decorrido do aumento exponencial da proporção de hemácias parasitadas ${ }^{5}$, acompanhado possivelmente da destruição aumentada de células não parasitadas por estresse oxidativo gerado, de forma aguda, em resposta ao parasita ${ }^{26}$. No segundo (P. malariae), ela teria sido resultado da cronicidade da infecção. Em ambos, o papel inibidor de citocinas inflamatórias sobre a eritropoiese pode também estar envolvido ${ }^{27-29} \mathrm{e}$ a anemia aparece como consequência do retardo diagnóstico da malária.
Atualmente, com a automatização dos exames de hemograma e com a prática comum das emergências e unidades de prontoatendimento da utilização exclusiva de hematócrito e plaquetas para diagnóstico de FHD perde-se a oportunidade da realização da hematoscopia, importante para visualização dos aspectos das células sanguíneas, como o tipo de anemia, e também para a detecção de hemozoários. Tal realidade enfatiza a necessidade de treinamento de qualidade de profissionais de saúde capazes de evocar a possibilidade diagnóstica de malária ${ }^{30}$, e técnicos competentes na identificação do parasita no exame direto, mesmo em áreas não endêmicas.

Pouco se sabe sobre os fatores envolvidos na cadeia de transmissão da malária autóctone ${ }^{31-33}$ no Estado do Rio de Janeiro. A presença de macacos bugios da espécie Alouatta guariba ${ }^{34}$, de Anopheles (Kertesia) cruzi na região de Mata Atlântica do Rio de Janeiro e a presença de malária em indivíduos que entraram em área de mata fechada são fortes indícios de que há circulação de Plasmodium na região ${ }^{35}$. Inquéritos sorológicos nessa região são necessários e poderiam informar sobre a circulação de Plasmodium no estado.

As três evoluções clínicas que apresentamos aqui ilustram a importância da febre como sinal clínico de emergência médica, em indivíduos provenientes de áreas endêmicas de malária, sobretudo em viajantes provenientes de regiões como o continente africano e a bacia Amazônica. Por outro lado, enfatizamos o treinamento dos profissionais de saúde locais para a vigilância da malária, que poderia ajudar a reduzir a morbiletalidade da malária na região extra-Amazônica, e a necessidade de encaminhamento imediato de pacientes para uma unidade de referência de pronto diagnóstico e tratamento de malária, que pode salvar vidas.

\section{CONFLITO DE INTERESSE}

Os autores declaram não haver nenhum tipo de conflito de interesse no desenvolvimento do estudo.

\section{SUPORTE FINANCEIRO}

Este trabalho é parte da dissertação de mestrado de APC que teve bolsa de mestrado da FIOTEC. CTDRé Cientista do Nosso Estado da FAPERJ e Bolsista de Produtividade do CNPQ.

\section{REFERÊNCIAS}

1. Organização Mundial de Saúde. Malaria [internet]. Genebra: Organização Mundial de Saúde. 2009 - [atualização março de 2009; acesso 02 de janeiro 2010]. Disponível em: <http://www.who.int/mediacentre/factsheets/fs094/en/index.html>.

2. Ministério da Saúde, Secretaria de Vigilância em Saúde. Situação Epidemiológica da Malária no Brasil. Brasília: Ministério da Saúde; 2006.

3. Ministério da Saúde, Secretaria de Vigilância em Saúde. Situação Epidemiológica da Malária no Brasil. Brasília: Ministério da Saúde; 2007.

4. Bruce-Chwatt LJ. Essential Malariology. $2^{\text {nd }}$ ed. London: William Heinemamm Medical Books; 1985.

5. Suárez-Mutis MC, Martinez-Espinosa FE, Albuquerque BC. Malária. In Coura JR, editor. Dinâmica das doenças infecciosas e parasitárias. Rio de Janeiro: Guanabara Koogan; 2005. 2v. p. 833-858.

6. Krugstad DJ.Malária. In: Goldman L, Ausiello D, editors. Cecil Tratado de Medicina Interna. 22a ed. Rio de Janeiro: Guanabara Koogan; 2005. p. 2421-2426.

7. Ministério da Saúde. Secretaria de Vigilância em Saúde. Guia de Vigilância Epidemiológica. Série A. Normas e Manuais Técnicos. 6a ed. Brasília: Ministério da Saúde; 2005. 
8. Alves A, Martins A, Adolphsson S, Bockorny B, Carleti G, Cabral G, et al. Severe Imported Malaria. Case Report. Rev Bras Ter Intensiva 2007; 19:232-236.

9. Ministério da Saúde. Fundação Nacional da Saúde. Manual de Terapêutica da Malária. Brasília: Ministério da Saúde. Fundação Nacional da Saúde; 2001.

10. Ministério da Saúde. Secretaria de Vigilância em Saúde. Programa Nacional de Prevenção e Controle da Malária PNCM. Série C. Projetos, Programas e Relatórios. Brasília: Ministério da Saúde; 2003.

11. Ministério da Saúde. Secretaria de Vigilância em Saúde. Manual de diagnóstico laboratorial da malária. Série A. Normas e Manuais Técnicos. Brasília DF: Ministério da Saúde; 2005.

12. Ministério da Saúde. Secretária de Vigilância em Saúde. Guia Prático de Tratamento da Malária no Brasil. Diretoria Técnica de Gestão. Série A. Normas e Manuais Técnicos Brasília: Ministério da Saúde; 2008.

13. Cunha RV, Nogueira RMR. Dengue. In: Coura JR, editor. Dinâmica das doenças infecciosas e parasitárias. Rio de Janeiro: Guanabara Koogan; 2005. 2v. p.1767-1781.

14. Centers for Disease Control and Prevention. Dengue. [Acessado em 31 jan. 2010.] Disponível em: <http://www.cdc.gov/dengue/>.

15. Gluber DJ. Epidemic dengue/dengue hemorragic fever as a public health, social and economic problem in the $21^{\text {st }}$ century. Trends Microbiol 2002; 10:100-103.

16. Teixeira MG, Costa MC, Guerra Z, Barreto ML. Dengue in Brazil: situation 2001 and trends. Dengue Bull 2002; 26:70-76.

17. Ministério da Saúde. Portal da Saúde. [Acessado em 09 novembro 2009] Disponível em: <http://portal.saude.gov.br>.

18. Superintendência de Vigilância em Saúde. Coordenação de Vigilância Epidemiológica. Secretária de Estado de Defesa Civil-Rio de Janeiro. Relatório de casos de dengue; 2008. [Acessado em 10 de novembro de 2009]. Disponível em: <http://www.saude.rj.gov.br/>.

19. Cunha RV. Aspectos clínicos e epidemiológicos da infecção pelos vírus dengue em áreas endêmicas do Brasil. Rev Soc Bras Med Trop 1998; 31:409-410.

20. Brasil P, Guaraldo L. Boletim de Farmacovigilância. Unidade de Farmacovigilância do Centro de Vigilância Sanitária da Secretaria Estadual de Saúde e Defesa Civil do Rio de Janeiro. Julho de 2007, nº4.

21. Ministério da Saúde. Secretaria de Vigilância em Saúde. Manual de diagnóstico laboratorial da malária. Série A. Normas e Manuais Técnicos. Brasília DF: Ministério da Saúde; 2005.

22. Torres KL, Figueiredo DV, Zalis MG, Daniel-Ribeiro CT, Alecrim W, Ferreirada-Cruz MF. Standardization of a very specific and sensitive single PCR for detection of Plasmodium vivax in low parasitized individuals and its usefulness for screening blood donors. Parasitol Res 2006; 98:519-524.

23. Zalis MG, Ferreira-da-Cruz MF, Balthazar-Guedes HC, Banic DM, Alecrim W, Souza JM, et al. Malaria diagnosis: standardization of a polymerase chain reaction for the detection of Plasmodium falciparum parasites in individuals with low-grade parasitemia. Parasitol Res 1996; 82:612-616.

24. Oliveira-Ferreira J, Lacerda MVG, Brasil P, Ladislau JLB, Tauil P, Daniel-Ribeiro CT. Malaria in Brazil: an overview. Malar J 2010; 9:115.

25. Araujo CF, Lacerda MVG, Abdalla DSP, Lima ES. The role of platelet and plasma markers of antioxidant status and oxidative stress in thrombocytopenia among patients with vivax malaria. Mem Inst Oswaldo Cruz 2008; 103:517-521.

26. Jakeman GN, Saul A, Hogart WL, Collins WE. Anemia of acute malaria in nonimune patients primarily results from destruction of uninfected erytrocytes. Parasitology 1999; 119(Pt 2):127-133.

27. Kanjaksha-Ghosh K, Kinjalka-Ghosh K. Pathogenesis of anemia in malaria: a concise review. Parasitol Res 2007; 101:1463-1469.

28. Fernandes AAM, Carvalho LJM, Zanini GM, Ventura AMRS, Souza JM, Cotias PM, et al. Similar cytokine responses and degrees of anemia in patients with Plasmodium falciparum and Plasmodium vivax infections induce similar cytokine responses and similar degrees of anemia in an area of coexisting transmission in the Brazilian Amazon region. Clin Vaccine Immunol 2008; 15:650-658.

29. Chang KH, Stevenson MM. Malarial anaemia: mechanisms and implications of insufficient erythropoiesis during blood-stage malaria. Int J Parasitol 2004; 34:1501-1516.

30. Wanderley DMV, Silva RA, Andrade JCR. Aspectos epidemiológicos da malária no Estado de São Paulo, Brasil, 1983 a 1992. Rev Saude Publica 1994; 28:192-197.
31. Marques GRSM, Condino MLF, Serpa LLN, Cursino TVM. Aspectos epidemiológicos de malária autóctone na mata atlântica, litoral norte, Estado de São Paulo, 1985-2006. Rev Soc Bras Med Trop 2008; 41:386-389.

32. Cerutti-Junior C. Caracterização epidemiológica da malária autóctone do Espírito Santo. [Tese de doutorado]. [São Paulo]: Faculdade de Medicina da Universidade de São Paulo; 2007. 228p.

33. Cerutti-Junior C, Boulos M, Coutinho AF, Hatab MCLD, Falqueto A, Rezende $\mathrm{HR}$, et al. Epidemiologic aspects of the malaria transmission cycle in an area of very low incidence in Brazil. Malar J 2007; 6:33.

34. Duarte AM, Malaforte RS, Cerutti-Junior C, Curado I, Paiva BR, Maeda A, et al. Natural Plasmodium infections in Brazilian wild monkeys: Reservoirs for human infections? Acta Tropica 2008; 107:179-185.

35. Costa AP, Ferreira-da-Cruz MF, Bressan CS, Pedro RS, Valls RS, Daniel-Ribeiro CT, et al. Malária de Mata Atlântica no Estado do Rio de Janeiro. Rev Soc Bras Med Trop 2009; 42 (supl I):477. 